\title{
Knowledge of Postnatal Care among Confinement Ladies in Malaysia during COVID-19 Pandemic: A Pilot Study
}

\author{
Mohd Jidin NA ${ }^{1}$, Mohamad $\mathrm{M}^{1}$, Wan Mohd Kamaluddin $\mathrm{WNS}^{1}$, Abd Aziz $\mathrm{KH}^{2}$, \\ Jamani $\mathrm{NA}^{3}$ \\ ${ }^{1}$ Medical Student, Kulliyyah of Medicine, IIUM, Pahang; ${ }^{2}$ Department of Community \\ Medicine, Kulliyyah of Medicine, IIUM, Pahang; ${ }^{3}$ Department of Family Medicine, Kulliyyah \\ of Medicine, IIUM, Pahang \\ DOI: http://dx.doi.org/10.31344/ijhhs.v5i0.313
}

Introduction: Mothers and their newborn babies are vulnerable to illnesses and deaths not only during pregnancy and birth but also during postnatal period. Hiring a confinement lady during this period is believed to be beneficial since postpartum home care is crucial in preventing postpartum complications.

Objective: This study aims to evaluate the knowledge of postnatal care among confinement ladies in Malaysia and assess the effectiveness of an intervention workshop.

Methods: A quasi-experimental study was conducted among 65 confinement ladies residing in East Coast Malaysia. Among them, 36 ladies were purposely selected to participate in a workshop, while 29 ladies were selected using a snowball sampling method to be in the control group. A validated Malay translation of Knowledge on Postnatal Care for Mothers and Care of the Newborn Questionnaire was used. Data were analysed using IBM SPSS Statistics (Version 25). Multiple logistic regression was employed to determine factors associated with baseline knowledge. Repeated measure ANCOVA was used to measure the effectiveness of the intervention workshop.

Results: Overall, the mean (SD) age of the respondents was 46.23 (8.49) with mean (SD) number of children of 4.0 (2.0) and working experience as confinement ladies of 36.0 months (20.24). Majority (77.1\%) were married, from B40 group (91.4\%) and received at least secondary education $(77.1 \%)$. Confinement ladies who received secondary and tertiary education were found to have higher knowledge scores compared to those with primary education ( $\mathrm{p}=0.026$ and $\mathrm{p}=0.049$ respectively). There was a significant increase in knowledge of postnatal care scores in those attending the postnatal care workshop $(p<0.001)$

Conclusion: Education level plays an important role in determining the level of knowledge of a confinement lady. The workshop conducted was effective in improving the postnatal care knowledge among confinement ladies. Hence, more interventional programs should be held in the future.

Keywords: postnatal care, knowledge, confinement ladies, Malaysia 